\title{
Comparing quality of life and treatment
}

\section{satisfaction between patients on warfarin and direct oral anticoagulants: a cross-sectional study}

This article was published in the following Dove Press journal:

Patient Preference and Adherence

\author{
Diana Leh-Ching $\mathrm{Ng}^{\prime}$ \\ Gin-Gin Gan ${ }^{2}$ \\ Chee-Shee Chail \\ Kok-Han Chee ${ }^{2}$ \\ Kok-Leng $\operatorname{Tan}^{3}$ \\ Seng-Beng $\operatorname{Tan}^{2}$ \\ Ping-Chong Bee ${ }^{2}$
}

'Department of Medicine, Faculty of Medicine and Health Science, University Malaysia Sarawak, Kota Samarahan, Sarawak, Malaysia; ${ }^{2}$ Department of Medicine, Faculty of Medicine, University of Malaya, Kuala Lumpur, Malaysia;

${ }^{3}$ Department of Medicine, Faculty of Medicine, University Science Malaysia, Penang, Malaysia
Correspondence: Gin-Gin Gan Department of Medicine, Faculty of Medicine, University of Malaya, Kuala Lumpur 50603, Malaysia

Tel +60379492741

Fax +60379556936

Email gangg@ummc.edu.my
Introduction and aim: Patient quality of life (QOL) while on long-term oral anticoagulant therapy has been receiving greater attention in recent years due to the increase in life expectancy brought about by advances in medical care. This study aimed to compare the QOL, treatment satisfaction, hospitalization and bleeding rate in patients on long-term warfarin versus direct oral anticoagulants (DOAC).

Methods: This was a cross-sectional study of patients with non-valvular atrial fibrillation (NVAF) or venous thromboembolism (VTE) on long-term anticoagulant therapy attending the cardiology clinic and anticoagulation clinic of the University Malaya Medical Centre from July 1, 2016, to June 30, 2018. Patient QOL was assessed by using the Short Form 12 Health Survey (SF12), while treatment satisfaction was assessed by using the Perception of Anticoagulation Treatment Questionnaire 2 (PACT-Q2).

Results: A total of 208 patients were recruited; $52.4 \%$ received warfarin and $47.6 \%$ received DOAC. There was no significant difference in QOL between warfarin and DOAC based on SF12 (physical QOL, $P=0.083$; mental QOL, $P=0.665$ ). Nevertheless, patients in the DOAC group were significantly more satisfied with their treatment compared to the warfarin group based on PACT-Q2 $(P=0.004)$. The hospitalisation rate was significantly higher in the warfarin group than the DOAC group $(15.6 \%$ versus $3.0 \%, P=0.002)$. Clinically relevant minor bleeds and severe bleeding events were non-significantly higher in the warfarin group than the DOAC group (66.7\% versus $40.0 \%, P=0.069)$.

Conclusion: Compared to warfarin, treatment of NVAF and VTE with DOAC showed comparable QOL, higher treatment satisfaction, lesser hospitalization, and a non-significant trend toward fewer bleeding episodes.

Keywords: quality of life, treatment satisfaction, convenience, warfarin, direct oral anticoagulants

\section{Introduction}

Warfarin, a vitamin $\mathrm{K}$ antagonist, has been widely used for decades to treat or prevent stroke and systemic embolism in patients with atrial fibrillation (AF) or venous thromboembolism (VTE). Warfarin has narrow therapeutic index, which requires frequent international normalized ratio (INR) monitoring to prevent bleeding complications and to maintain therapeutic efficacy. The target range of INR may vary depending on the indications for anticoagulation. ${ }^{1,2}$ The use of warfarin is challenging, as there is considerable interpatient variability in the daily maintenance dose of warfarin. In addition, numerous foods and drugs as well as alcohol are 
known to interact with the metabolism of warfarin. ${ }^{3}$ Genetic variation in the metabolism of warfarin also has been shown to affect its efficacy. ${ }^{4}$ Despite these challenges, warfarin is still a commonly used oral anticoagulant, mainly due to its affordability and availability.

Direct oral anticoagulants (DOAC) such as dabigatran, rivaroxaban, apixaban and edoxaban have been developed to overcome the challenges of using warfarin. Dabigatran is a direct inhibitor of Factor IIa, while rivaroxaban, apixaban and edoxaban are direct inhibitors of Factor Xa. ${ }^{5}$ The advantages of DOAC include simple dosing without the need for monitoring, no need for dietary restriction, fewer drug-drug interactions, a rapid onset of action and a shorter half-life which allows for the possibility of uncomplicated switching or bridging therapy. ${ }^{6}$ Nevertheless, these drugs are expensive and require strict adherence to the treatment due to its fast offset of action. Being newer oral anticoagulants, there is still limited evidence on the usage of DOAC in conditions such as antiphospholipid syndrome and valvular heart disease, nor are the risks involved with prosthetic heart valves and susceptible populations clearly known. ${ }^{5}$ The reversal agents for these newer agents are not widely available and are more expensive than using vitamin $\mathrm{K}$ for warfarin.

Patient quality of life (QOL) while on long-term oral anticoagulant therapy has been receiving greater attention in recent years due to the increase in life expectancy brought about by advances in medical care. QOL is defined as individual satisfaction or happiness with an aspect of life that is affected by their health either in physical (PF), mental, emotional or social functioning (SF). ${ }^{7}$ Treatment with an oral anticoagulant may affect patient QOL because it requires a change of lifestyle, increased risk of bleeding and does not provide objective symptomatic relief. Higher satisfaction with regard to anticoagulant treatment is associated with better treatment adherence and therefore improved QOL. ${ }^{8,9}$ Treatment satisfaction is defined as an individual's rating of important attributes of the process and outcomes of his or her treatment experience, which involve the interaction of expectation, preference and satisfaction. ${ }^{10,11}$

To date, published data that compare QOL and treatment satisfaction in patients on long-term warfarin versus DOAC are limited. Therefore, the primary objectives of this study were to compare QOL and treatment satisfaction in patients on long-term warfarin versus DOAC. The secondary objectives were to compare hospitalization and bleeding rates between the two groups.

\section{Methodology}

\section{Study design}

This was a cross-sectional study comparing QOL and treatment satisfaction in patients receiving long-term warfarin or DOAC attending the cardiology clinic and anticoagulation clinic of University Malaya Medical Centre (UMMC) from 1st July 2016 to 30th June 2018. All patients were aged 18 years and above, and had been treated with the same oral anticoagulant for at least 6 months. Patients were categorized as receiving DOAC if they were treated with dabigatran, rivaroxaban or apixaban. Indications for warfarin and DOAC in these patients include underlying non-valvular AF (NVAF) or VTE. Patients were excluded in the case of underlying issues such as mechanical heart valves, valvular heart disease, malignancy, concomitant anti-platelet therapy, cross-over from warfarin to DOAC or vice-versa, hospital admission within 1 month of the interview due to any cause other than complications with their oral anticoagulant, or cognitive/visual impairments that restricted patients from answering questionnaires independently. Patients who were treated with warfarin but had an incomplete INR record were also excluded. This study was approved by the Ethics Committee of UMMC. Written informed consent was obtained from all participants.

\section{Data collection and questionnaires}

Eligible patients were identified from the registry of hospital and approached by investigators on the day of their respective clinic visit. Patient demographic and clinical data were obtained from their electronic medical records and face-to-face interviews.

For patients on warfarin, their INR readings collected over the past 6 months to 1 year were recorded. Patient INR values were tested using the CoaguChek XS system (Roche Diagnostics, Mannheim, Germany), a point-of-care device at the clinic. Time in therapeutic range (TTR), which is defined as the duration of time in which the patient's INR values were within a desired range, was calculated using the Rosendaal method. ${ }^{12}$ The INR target range was defined according to American College of Chest Physician guidelines, in which the optimum INR target ranges from 2.0 to 3.0 . For those with specific VTE cases, the INR target range was 2.5-3.5. ${ }^{1,2}$ The INR in the first 6 weeks after the initiation of warfarin was excluded from the calculation of TTR. In this study, the cut-off point for good TTR was set at $60 \%$ because of any 
value that $<58 \%$ does not confer a greater clinical benefit than dual anti-platelet therapy. ${ }^{13}$

In assessing QOL and treatment satisfaction, the patients were instructed to answer the Short Form 12v2 Health Survey (SF12v2) and Perception of Anticoagulation Treatment Questionnaire 2 (PACT-Q2) questionnaires independently with minimal assistance from the investigators. The patients could choose to answer the original English version, validated Malay version or validated Chinese version. ${ }^{14-16}$ SF12v2 assesses patient QOL in eight domains, namely PF, role physical, bodily pain, general health perceptions, vitality, SF, role emotional and mental health. The physical component summary (PCS) and mental component summary (MCS) were calculated from the eight domains using the Quality Metric's Health Outcome ${ }^{\text {TM }}$ Scoring Software 5.0. A higher score corresponds to better health state in patients. PACT-Q2 consists of 20 items divided into three domains, namely " $\mathrm{B}$ " for convenience, " $\mathrm{C}$ " for burden of disease and " $\mathrm{D}$ " for treatment and anticoagulation treatment satisfaction. ${ }^{17,18}$ The convenience score was the sum of all the items that were inverted in domains B and C (each item score $=6$ minus the initial score), while the satisfaction score was the sum of the item scores in domain D. A higher score corresponds to greater convenience or satisfaction regarding the anticoagulant. For patients receiving DOAC, item B5 of PACT-Q2, i.e., "certain food to be avoided while taking anticoagulant," was replaced by "Is it difficult for you to take your tablet during meals, as recommended?" because DOAC do not interact with food. ${ }^{8,19}$ Besides, DOAC are recommended to be taken with food to increase absorption. ${ }^{20}$ Therefore, the original PACT-Q2 scoring was only used in patients receiving warfarin, while a modified version of scoring was used in patients receiving DOAC.

Hospitalization was recorded when the reason for admission was attributed to complications with the anticoagulant such as bleeding or recurrent thromboembolism. Patient bleeding history was defined according to the International Society on Thrombosis and Haemostasis bleeding scale. $^{21,22}$

\section{Analysis}

Categorical variables were expressed as percentages, while continuous variables were expressed as the mean $\pm \mathrm{SD}$ or median with interquartile range. For categorical variables, the difference between anticoagulant groups was compared using the chi-squared test or Fisher's exact test. For continuous variables, the difference between groups was compared by using independent sample $t$-test or the Mann-Whitney U test. The difference in QOL and treatment satisfaction between anticoagulant groups with age and treatment duration as covariates was analyzed by ANCOVA. A $P<0.05$ was considered statistically significant in this study. Statistical Package for the Social Sciences (SPSS for Windows version 25.0, SPSS Inc., Chicago, IL, USA) was used for the statistical analysis.

\section{Results}

\section{Patient demographics and clinical data}

A total of 208 patients were recruited. The demographic characteristics are shown in Table $1.52 .4 \%$ of the patients were taking warfarin and $47.6 \%$ of patients were taking DOAC. The mean TTR was $54.9 \pm 24.8 \%$, with only $45.0 \%$ of patients achieving a good TTR. The warfarin group was significantly younger (mean age $61.3 \pm 15.9$ versus 69.1 \pm 12.1 years, $P<0.001$ ), and had a significantly longer treatment duration (mean duration $8.5 \pm 7.0$ versus $3.2 \pm 2.0$ years, $P<0.001)$ compared to the DOAC group. The DOAC group had significantly more assisted funding from the government ( $44.4 \%$ versus $20.2 \%, P<0.001)$, underlying NVAF $(88.9 \%$ versus $67.9 \%, P<0.001)$ and polypharmacy $(49.5 \%$ versus $32.1 \%, P=0.003)$ compared to the warfarin group. There was no significant difference in the distribution of gender, ethnicity, marital status, education level, diet preference, alcohol intake and comorbidities between the two groups.

\section{QOL}

The overall QOL of the warfarin and DOAC groups was not significantly different. There was no significant difference in the score of PCS $(45.0 \pm 10.2$ versus $42.7 \pm 9.7$, $P=0.083), \operatorname{MCS}(52.7 \pm 8.5$ versus 52.3 $\pm 8.1, P=0.665)$ and each domain of SF12v2 $(P=0.055-0.960)$ between the warfarin and DOAC groups (Table 2). When adjusted for age and treatment duration, the scores for PCS $(44.3 \pm 0.9$ versus $43.4 \pm 1.0, P=0.502 ; 45.1 \pm 1.0$ versus $42.6 \pm 1.1$, $P=0.105)$, MCS (53.0 \pm 0.8 versus 51.9 $\pm 0.8, P=0.365$; $52.5 \pm 0.8$ versus $52.5 \pm 0.9, P=0.982)$ and each domain of SF12v2 $(P=0.122-0.979 ; P=0.220-0.968)$ were not significantly different between the warfarin and DOAC groups.

\section{Treatment satisfaction (convenience score)}

The overall convenience score was not significantly different between the warfarin and DOAC groups $(79.8 \pm 16.9$ 
Table I Demographic characteristics of patients on oral anticoagulants

\begin{tabular}{|c|c|c|c|c|}
\hline \multicolumn{2}{|l|}{ Characteristics } & \multicolumn{2}{|c|}{ Anticoagulant type (n, \%) } & \multirow{3}{*}{\begin{tabular}{|l|} 
P-value \\
$<0.001$ \\
\end{tabular}} \\
\hline & & \multirow{2}{*}{$\begin{array}{l}\text { Warfarin } 109(52.4) \\
61.3 \pm 15.9\end{array}$} & \multirow{2}{*}{$\frac{\text { DOACs } 99(\mathbf{4 7 . 6 )}}{69.1 \pm \mid 2.1}$} & \\
\hline Age (mean $\pm S D)$ & Years & & & \\
\hline Gender (n, \%) & $\begin{array}{l}\text { Male } \\
\text { Female }\end{array}$ & $\begin{array}{l}55(50.5) \\
54(49.5)\end{array}$ & $\begin{array}{l}52(52.5) \\
47(47.5)\end{array}$ & 0.766 \\
\hline Partner status (n, \%) & $\begin{array}{l}\text { No partner } \\
\text { With partner }\end{array}$ & $\begin{array}{l}35(32.1) \\
74(67.9)\end{array}$ & $\begin{array}{l}35(35.4) \\
64(64.6)\end{array}$ & 0.621 \\
\hline Ethnicity (n, \%) & $\begin{array}{l}\text { Malay } \\
\text { Chinese } \\
\text { Indian } \\
\text { Others }\end{array}$ & $\begin{array}{l}39(35.8) \\
63(57.8) \\
5(4.6) \\
2(1.8)\end{array}$ & $\begin{array}{l}37(37.4) \\
45(45.5) \\
13(13.1) \\
4(4.0)\end{array}$ & 0.078 \\
\hline Education (n, \%) & $\begin{array}{l}\text { None } \\
\text { Primary } \\
\text { Secondary } \\
\text { College/tertiary }\end{array}$ & $\begin{array}{l}6(5.5) \\
27(24.8) \\
54(49.5) \\
22(20.2)\end{array}$ & $\begin{array}{l}7(7.1) \\
19(19.2) \\
44(44.4) \\
29(29.3)\end{array}$ & 0.395 \\
\hline Occupation (n, \%) & $\begin{array}{l}\text { Unemployed } \\
\text { Government dependent/pensioner } \\
\text { Private }\end{array}$ & $\begin{array}{l}57(52.3) \\
22(20.2) \\
30(33.2)\end{array}$ & $\begin{array}{l}44(44.4) \\
44(44.4) \\
\text { II }(11.1)\end{array}$ & $<0.001$ \\
\hline Diet $(n, \%)$ & $\begin{array}{l}\text { Non-vegetarian/vegan } \\
\text { Vegetarian/vegan }\end{array}$ & $\begin{array}{l}105(96.3) \\
4(3.7)\end{array}$ & $\begin{array}{l}92(92.9) \\
7(7.1)\end{array}$ & 0.274 \\
\hline Alcohol (n, \%) & $\begin{array}{l}\text { No } \\
\text { Yes }\end{array}$ & $\begin{array}{l}103(94.5) \\
6(3.8)\end{array}$ & $\begin{array}{l}98(99.0) \\
1(1.0)\end{array}$ & 0.073 \\
\hline Comorbidities (n, \%) & $\begin{array}{l}\leq 2 \\
>2\end{array}$ & $\begin{array}{l}69(63.3) \\
40(36.7)\end{array}$ & $\begin{array}{l}53(53.5) \\
46(46.5)\end{array}$ & 0.153 \\
\hline Other drugs (n, \%) & $\begin{array}{l}\text { None } \\
<5 \\
\geq 5\end{array}$ & $\begin{array}{l}11(10.1) \\
63(57.8) \\
35(32.1)\end{array}$ & $\begin{array}{l}\text { I (I.0) } \\
49(49.5) \\
49(49.5)\end{array}$ & 0.003 \\
\hline Indication for anticoagulation (n, \%) & $\begin{array}{l}\text { NVAF } \\
\text { VTE }\end{array}$ & $\begin{array}{l}74(67.9) \\
35(32.1)\end{array}$ & $\begin{array}{l}88(88.9) \\
\text { II (II.I) }\end{array}$ & $<0.001$ \\
\hline Duration of therapy (mean $\pm S D$ ) & Years & $8.5 \pm 7.0$ & $3.2 \pm 2.0$ & $<0.001$ \\
\hline TTR $($ mean $\pm S D)$ & $\%$ & $54.9 \pm 24.8$ & - & - \\
\hline Good TTR (n, \%) & $\begin{array}{l}\text { Yes } \\
\text { No } \\
\text { Reasons: } \\
\text { - Above therapeutic range } \\
\text { - Above and below therapeutic range } \\
\text { - Below therapeutic range }\end{array}$ & $\begin{array}{l}49(45.0) \\
60(55.0) \\
3(5.0) \\
46(76.7) \\
11(18.3)\end{array}$ & - & - \\
\hline
\end{tabular}

Abbreviations: DOACs, direct oral anticoagulants; NVAF, non-valvular atrial fibrillation; VTE, venous thromboembolism; TTR, time in therapeutic range.

versus $82.7 \pm 16.7, P=0.229$ ) (Table 3 ). After adjustment for age and treatment duration, the convenience scores of the warfarin and DOAC groups remained not significantly different $(80.7 \pm 1.6$ versus $81.7 \pm 1.7, P=0.658 ; 80.1$ versus 1.7 versus $82.3 \pm 1.8, P=0.410$ ).
In the subgroup analysis, the DOAC group had significantly better convenience scores than the warfarin group on item B5 - difficulties in avoidance of certain food $(4.5 \pm 0.9$ versus $3.9 \pm 1.2, P<0.001)$ and item B7 - difficulties regarding daily life $(4.3 \pm 1.0$ versus $4.0 \pm 1.1, P=0.047)$. After 


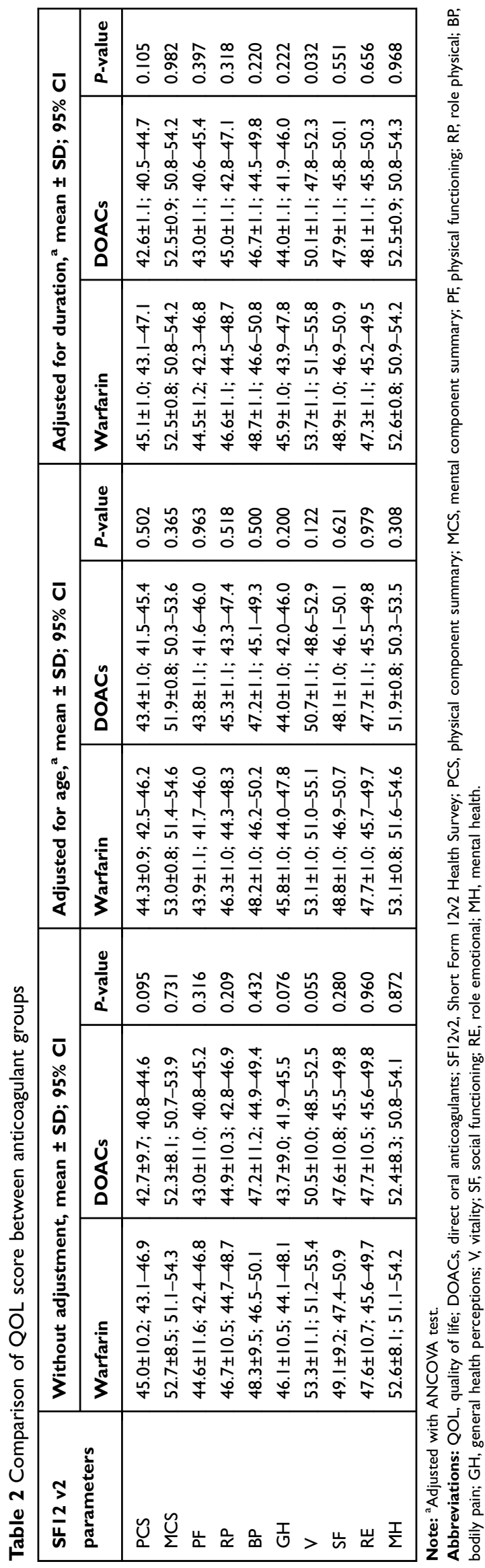

adjustment for age and treatment duration, only the difference in item B5 remained significant when comparing the DOAC group and the warfarin group ( $4.5 \pm 0.1$ versus \pm 3.9 $\pm 0.1, P=0.001 ; 4.5 \pm 0.1$ versus $\pm 3.9 \pm 0.1, P<0.001)$.

\section{Treatment satisfaction (satisfaction score)}

The overall satisfaction score was significantly higher in the DOAC group compared to the warfarin group (73.5 \pm 12.8 versus $68.7 \pm 11.3, P=0.004$ ) (Table 3 ). The satisfaction score remained significantly higher in the DOAC group compared to the warfarin group after adjusting for age $(73.7 \pm 1.2$ versus $68.5 \pm 1.2, P=0.004)$ and treatment duration (73.2 \pm 1.3 versus $69.0 \pm 1.2, P=0.026)$.

In the subgroup analysis, the satisfaction score of the DOAC group was higher than that of the warfarin group for each item except item D2 - decrease in symptoms. A significantly higher satisfaction score was recorded for item D3 - experience with side effects $(4.1 \pm 1.1$ versus $3.4 \pm 0.8, P<0.001$ ), which remained significantly higher even after adjusting for age (4.0 0.1 versus $3.5 \pm 0.1$, $P<0.001)$ and treatment duration $(4.0 \pm 0.1$ versus $3.5 \pm 0.1$, $P=0.001)$. The DOAC group also recorded significantly a higher satisfaction score for item D6 - satisfaction with treatment form ( $4.3 \pm 0.7$ versus $4.1 \pm 0.5, P=0.022)$, but the difference was not significant after adjusting for age $(P=0.067)$ and treatment duration $(P=0.052)$. Finally, the score for item D7 - overall satisfaction was significantly higher in the DOAC group than in the warfarin group after adjusting for age (4.2 \pm 0.1 versus $4.0 \pm 0.1, P=0.041)$.

\section{Hospitalization and complications}

The hospitalization rate was significantly higher in the warfarin group than in the DOAC group $(15.6 \%$ versus $3.0 \%, P=0.002)$ (Table 4). The main reason for hospitalization in the warfarin group was bleeding (70.6\%), while the main reason in the DOAC group was recurrent thrombosis (66.7\%). However, there were no significant differences in the complications of anticoagulants such as overall bleeding events $(24.8 \%$ versus $20.2 \%, P=0.431)$ or thromboembolism events $(5.5 \%$ versus $3.0 \%, P=0.381$ ). Among 47 patients with bleeding events, the clinically relevant minor bleeds and severe bleeding events were non-significantly higher in the warfarin group than in the DOAC group $(66.7 \%$ versus $40.0 \%, P=0.069)$.

\section{Discussion}

In this cross-sectional study, patients who received DOAC were significantly more satisfied with their treatment, but there was no difference in QOL or overall convenience 


\begin{tabular}{|c|c|c|c|c|c|c|c|c|c|c|c|c|c|c|c|c|}
\hline \multirow{3}{*}{ 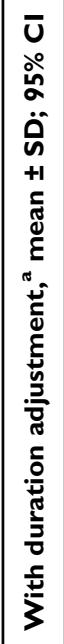 } & 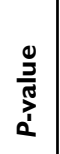 & $\frac{O}{\dot{t}}$ & & \begin{tabular}{|c}
0 \\
$\infty$ \\
$\infty$ \\
0 \\
0
\end{tabular} & 亗 & ণั่ & $\begin{array}{l}\sigma \\
\hat{\alpha} \\
o\end{array}$ & $\begin{array}{l}\overline{8} \\
\dot{0} \\
\mathrm{v}\end{array}$ & 惫 & $\frac{8}{0}$ & $\frac{m}{0}$ & $\frac{\text { I }}{0}$ & $\stackrel{\infty}{\stackrel{\infty}{0}}$ & $\frac{n}{m}$ & $\begin{array}{l}\text { ते } \\
\text { ర్ }\end{array}$ & 苂 \\
\hline & 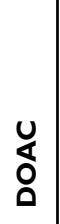 & 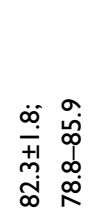 & & 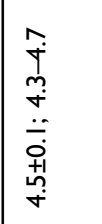 & $\begin{array}{l}0 \\
\stackrel{+}{+} \\
\stackrel{+}{+} \\
\dot{+} \\
\dot{+} \\
\dot{+} \\
\dot{+}\end{array}$ & 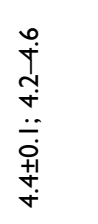 & 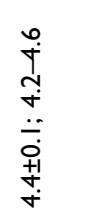 & 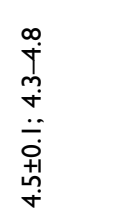 & 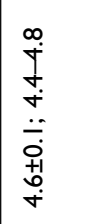 & 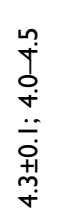 & 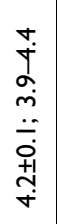 & $\begin{array}{l}\infty \\
\dot{J} \\
+ \\
+ \\
\dot{-} \\
\dot{0} \\
+1 \\
0 \\
+\end{array}$ & $\begin{array}{l}m \\
\dot{+} \\
\infty \\
\ddot{m} \\
\ddot{\bar{c}} \\
\dot{+1} \\
\dot{+}\end{array}$ & 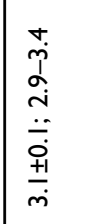 & 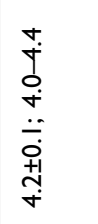 & 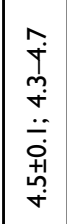 \\
\hline & 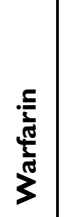 & 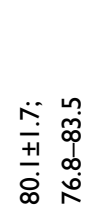 & & \begin{tabular}{|c}
$\hat{f}$ \\
$\dot{J}$ \\
$\dot{+}$ \\
$\dot{+}$ \\
$\dot{+1}$ \\
$\dot{+}$ \\
$\dot{\sigma}$
\end{tabular} & 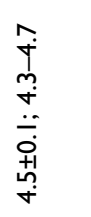 & $\begin{array}{l}+ \\
\dot{+} \\
0 \\
\dot{+} \\
\dot{+} \\
\dot{+} \\
+ \\
\dot{+}\end{array}$ & 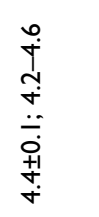 & $\begin{array}{c}\bar{y} \\
\stackrel{\dot{y}}{m} \\
\dot{\bar{c}} \\
\dot{+} \\
\dot{m} \\
\dot{m}\end{array}$ & 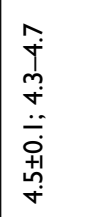 & 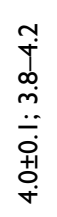 & 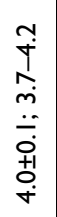 & 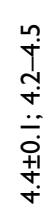 & 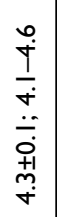 & 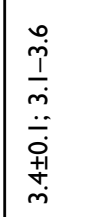 & 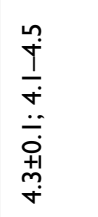 & 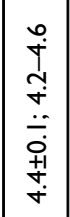 \\
\hline \multirow{3}{*}{ 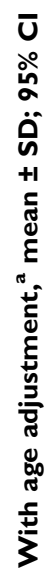 } & 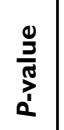 & $\begin{array}{l}\infty \\
0 \\
0 \\
0 \\
0\end{array}$ & & 亲 & $\frac{\circ}{0}$ & $\stackrel{\substack{m \\
\\
0}}{0}$ & $\begin{array}{l}\text { ồ } \\
\text { ô }\end{array}$ & $\bar{o}$ & 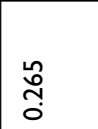 & $\frac{}{\overline{0}}$ & $\frac{\tilde{0}}{0}$ & $\begin{array}{l}\stackrel{0}{f} \\
0\end{array}$ & $\frac{\grave{T}}{0}$ & 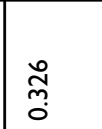 & 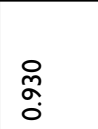 & 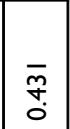 \\
\hline & 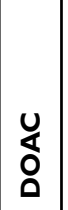 & 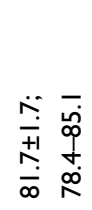 & & $\begin{array}{c}0 \\
\stackrel{0}{+} \\
m \\
\stackrel{+}{+} \\
\ddot{\overline{+}} \\
\stackrel{+}{+1} \\
\stackrel{+}{+}\end{array}$ & $\begin{array}{l}\stackrel{n}{+} \\
\underset{+}{+} \\
\underset{+}{*} \\
\ddot{\overline{0}} \\
\stackrel{+}{+1} \\
\stackrel{+}{+}\end{array}$ & 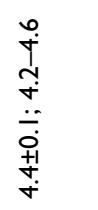 & 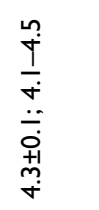 & 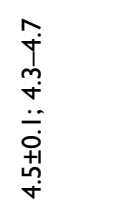 & 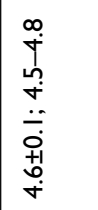 & 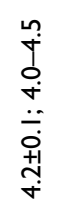 & $\begin{array}{c}\stackrel{+}{+} \\
\dot{I} \\
\dot{+} \\
\ddot{\bar{t}} \\
\stackrel{+}{+} \\
\stackrel{+}{+}\end{array}$ & 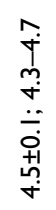 & $\begin{array}{c}m \\
\stackrel{+}{+} \\
\infty \\
m \\
\ddot{\bar{c}} \\
\dot{+} \\
\dot{+}\end{array}$ & 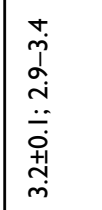 & $\begin{array}{l}\stackrel{\forall}{+} \\
\dot{+} \\
\dot{+} \\
\ddot{\bar{O}} \\
\dot{+} \\
\dot{+} \\
\dot{T}\end{array}$ & 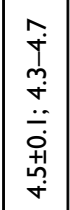 \\
\hline & 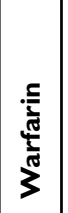 & 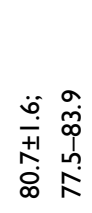 & & $\begin{array}{c}\sigma \\
\dot{I} \\
\dot{+} \\
\dot{+} \\
\ddot{0} \\
\dot{0} \\
+1 \\
0 \\
\dot{+}\end{array}$ & $\begin{array}{l}\hat{+} \\
\dot{+} \\
\dot{+} \\
\ddot{\circ} \\
+1 \\
\dot{+} \\
\dot{+}\end{array}$ & 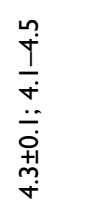 & 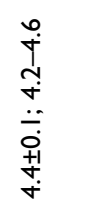 & $\begin{array}{l}\overline{\dot{y}} \\
\hat{m} \\
\ddot{\overline{\dot{c}}} \\
\dot{+} \\
\dot{m}\end{array}$ & 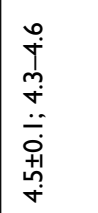 & 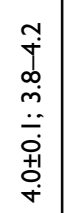 & $\begin{array}{c}\stackrel{\sim}{+} \\
\stackrel{+}{+} \\
\hat{m} \\
\ddot{\bar{O}} \\
+ \\
\dot{+} \\
\dot{+}\end{array}$ & 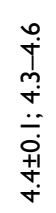 & 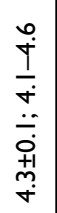 & $\begin{array}{c}0 \\
\stackrel{p}{1} \\
\frac{1}{m} \\
\ddot{\bar{c}} \\
\dot{+} \\
\dot{+1} \\
m\end{array}$ & $\begin{array}{l}\stackrel{+}{+} \\
\dot{+} \\
\dot{+} \\
\ddot{\bar{O}} \\
\dot{+} \\
\dot{+}\end{array}$ & 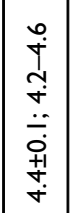 \\
\hline \multirow{3}{*}{ 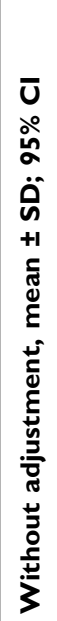 } & 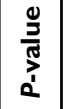 & స్తి & & $\begin{array}{l}R \\
\text { o. } \\
0\end{array}$ & 栥 & $\begin{array}{l}\bar{\alpha} \\
\text { Oे }\end{array}$ & $\begin{array}{c}\alpha \\
\stackrel{\alpha}{\alpha} \\
0 \\
0\end{array}$ & $\begin{array}{l}\bar{o} \\
\dot{0} \\
\mathrm{v}\end{array}$ & $\frac{2}{0}$ & $\begin{array}{l}\hat{y} \\
0 \\
0\end{array}$ & $\begin{array}{l}\text { Uू. } \\
\text { Oें }\end{array}$ & 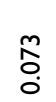 & $\begin{array}{l}q \\
0 \\
0 \\
0\end{array}$ & \begin{tabular}{|l}
8 \\
\\
0 \\
0
\end{tabular} & $\begin{array}{l}0 \\
\text { ర్ } \\
0 \\
0\end{array}$ & 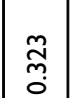 \\
\hline & 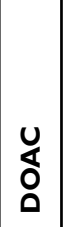 & 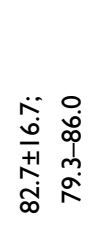 & & 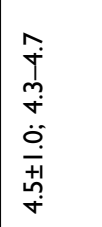 & 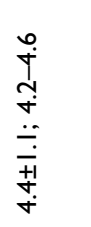 & 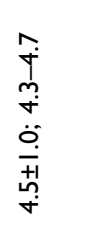 & 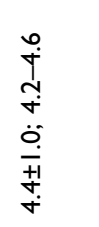 & 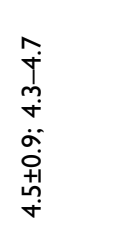 & 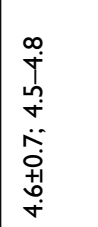 & 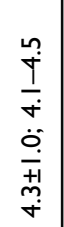 & 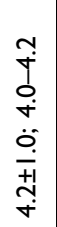 & $\begin{array}{l}\hat{y} \\
\dot{y} \\
\dot{j} \\
\dot{0} \\
0 \\
\dot{0} \\
0 \\
\dot{\sigma}\end{array}$ & $\begin{array}{l}m \\
\dot{y} \\
\infty \\
m \\
\dot{m} \\
\dot{m} \\
\dot{+} \\
\dot{+} \\
\dot{r}\end{array}$ & 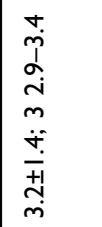 & 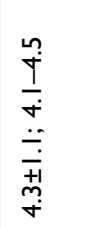 & 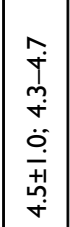 \\
\hline & 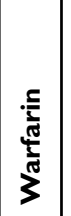 & 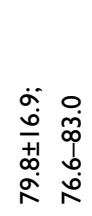 & & 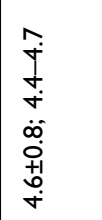 & 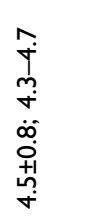 & 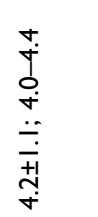 & 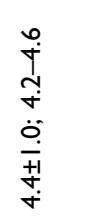 & 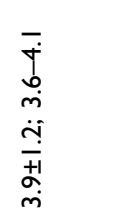 & 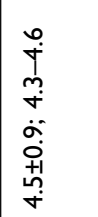 & 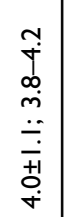 & $\begin{array}{l}\bar{j} \\
\hat{T} \\
m \\
\dot{T} \\
\dot{T} \\
+1 \\
\dot{m} \\
\dot{m}\end{array}$ & 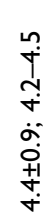 & 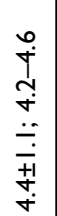 & 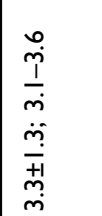 & 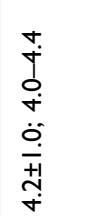 & 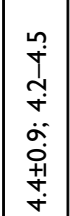 \\
\hline 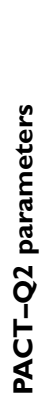 & & 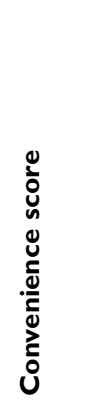 & 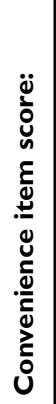 & 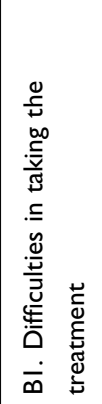 & 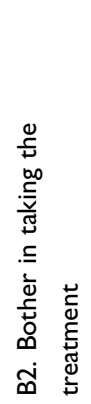 & 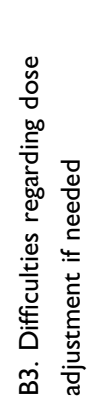 & 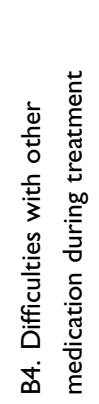 & 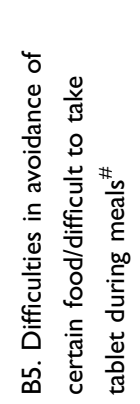 & 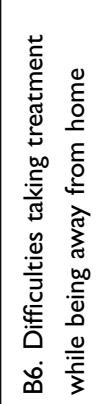 & 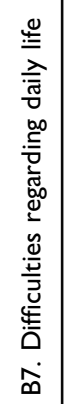 & 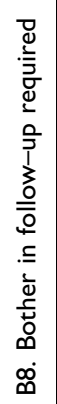 & 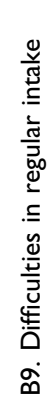 & 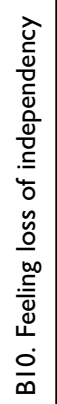 & 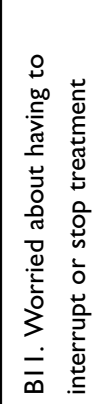 & 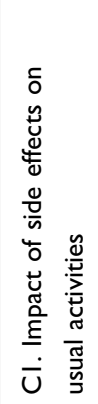 & 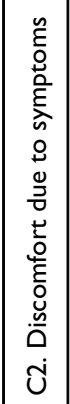 \\
\hline
\end{tabular}




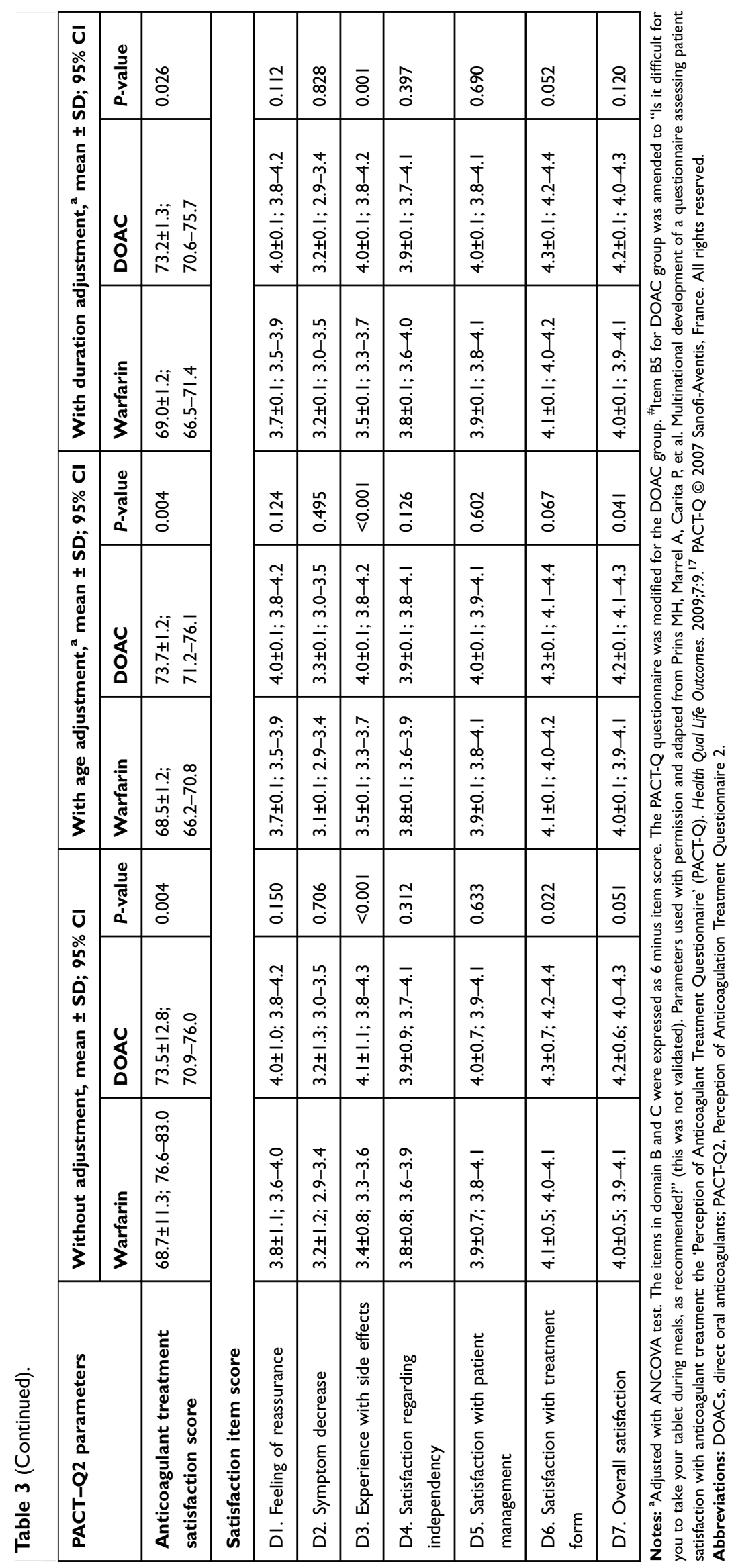


when compared to those receiving warfarin. The sub-analysis of the satisfaction domain demonstrated that patients receiving DOAC were more satisfied with the drug's side effects as they were significantly less severe than what they expected. This was supported by the finding of a significantly lower hospitalization rate as well as fewer clinically relevant minor bleeds and severe bleeding events among this group in this study. Additionally, patients receiving DOAC were more confident in their treatment, as well as more satisfied with the drug form and their clinical follow-up. This could be due to the advantages of DOAC as a newer generation of oral anticoagulant, with simpler dosing the absence of frequent blood monitoring, therefore fewer blood tests and clinic visits. Despite no difference in the total convenience score, patients receiving DOAC also reported significantly more convenience in their food intake compared to those receiving warfarin. DOAC should be taken with food, while warfarin requires control on a high vitamin K diet. Patients receiving DOAC and warfarin did not report significantly different QOL because anticoagulants neither provide objective symptomatic relief nor decrease symptoms. Moreover, QOL assessed beyond 6 months of treatment initiation allowed the patients to adapt to their respective treatments.

Another important finding highlighted in this study was that patients receiving warfarin appeared to have more clinically relevant minor bleeds and severe bleeding events. The lack of statistical significance could be due to the small number of patients who suffered from bleeding complications. The majority of patients in this study who failed to achieve a good TTR had INR above the therapeutic range, which could be a risk for bleeding events.

Monz et al reported the only QOL comparison for long-term warfarin versus DOAC in a clinical trial, in which there was no significant difference in the EuroQOL Instrument (EQ-5D) of AF patients in the Randomized Evaluation of Long-term Anticoagulant Therapy (RELY) sub-study. ${ }^{23}$ Similarly, Benzimra et al, Contreras et al and Alegret et al reported no significant differences in QOL between AF patients receiving longterm warfarin or DOAC. ${ }^{19,24,25}$ QOL was assessed by EuroQOL Instrument 3 levels (EQ-5D-3L) in the former two studies, while the Sawicki questionnaire was used in the latter study. Recently, Keita et al reported the only reallife comparison of QOL between patients receiving longterm warfarin versus DOAC in VTE, which failed to show a significant difference in the EQ-5D. ${ }^{8}$ Several studies have shown that patients on warfarin required at least 3 months to adapt to their treatment. ${ }^{23,25-27}$ Their limitations during the initial period may include a higher number of clinic visits, frequent blood tests, abrupt diet modifications and difficulty in achieving the expected INR. More than 6 months after treatment initiation, patients should have adapted to the anticoagulant treatment, which may explain the lack of difference in QOL between the oral anticoagulants in these studies. Another real-life study by Balci et al, however, reported a significantly better QOL assessed by Short Form-36 (SF36), a lower Hospital Anxiety

Table 4 Hospitalization, bleeding and systemic embolism between anticoagulant groups

\begin{tabular}{|c|c|c|c|c|}
\hline \multicolumn{2}{|l|}{ Characteristics } & \multicolumn{2}{|c|}{ Anticoagulant type (n, \%) } & \multirow[t]{2}{*}{$P$-value } \\
\hline & & $\begin{array}{l}\text { Warfarin } \\
109(52.4)\end{array}$ & $\begin{array}{l}\text { DOACs } \\
99(47.6)\end{array}$ & \\
\hline \multirow[t]{2}{*}{ Hospitalization (n, \%) } & No & $92(84.4)$ & $96(97.0)$ & \multirow[t]{2}{*}{0.002} \\
\hline & $\begin{array}{l}\text { Yes } \\
\text { Bleeding } \\
\text { Thrombosis }\end{array}$ & $\begin{array}{l}17(15.6) \\
12(70.6) \\
5(29.4)\end{array}$ & $\begin{array}{l}3(3.0) \\
1(33.3) \\
2(66.7)\end{array}$ & \\
\hline \multirow[t]{4}{*}{ Bleeding severity $(\mathrm{n}, \%)$} & No & $82(75.2)$ & $79(79.8)$ & \multirow[t]{4}{*}{0.270} \\
\hline & Minor & $9(8.3)$ & $12(12.1)$ & \\
\hline & Non-major, clinically relevant & $15(13.8)$ & $7(7.1)$ & \\
\hline & Major & $3(2.8)$ & $\mathrm{I}(\mathrm{I} .0)$ & \\
\hline \multirow[t]{2}{*}{ Systemic embolism (n, \%) } & No & $103(94.5)$ & $96(97.0)$ & \multirow[t]{2}{*}{0.381} \\
\hline & Yes & $6(5.6)$ & $3(3.0)$ & \\
\hline
\end{tabular}


Depression scale score, a lower hospitalization rate, as well as a lower incidence of any type of bleeding event in $\mathrm{AF}$ patients receiving long-term DOAC compared to warfarin. $^{28}$ The DOAC group in this study had been previously treated with warfarin, which allowed these patients to compare both anticoagulant therapies and may have led to a bias in self-reported QOL.

Concerning treatment satisfaction, Prins et al reported significantly better satisfaction in a subgroup of patients receiving DOAC versus enoxaparin/warfarin in the EINSTEIN pulmonary embolism trial. ${ }^{29}$ Treatment satisfaction in this study was assessed by the Anti-Clot Treatment Scale (ACTS) and the Treatment Satisfaction Questionnaire for Medication II. Cano et al also reported a significantly better ACTS burden score and numerically better ACTS benefit score in patients receiving DOAC versus the standard anticoagulant in the XA inhibition with rivaroxaban for Long-term and Initial Anticoagulation in venous thromboembolism (XALIA) study. ${ }^{30}$ In other real-world studies, Willich et al, Keita et al and Benzimra et al reported significantly better satisfaction and convenience among patients receiving DOAC versus warfarin for VTE or AF using PACT-Q2. ${ }^{8,19,31}$ Recently, Contreras et al also reported a similar result that favored DOAC in their AF patients based on ACTS and the Satisfaction Questionnaire. ${ }^{24}$ In short, significantly better treatment satisfaction among patients receiving long-term DOAC compared to warfarin has been consistently highlighted in existing clinical trials and real-life studies.

The present study concludes that DOAC is a better option for oral anticoagulant therapy in patients with NVAF or VTE because of greater satisfaction, more convenience regarding food intake, less frequent hospitalization, as well as fewer clinically relevant minor bleeds and severe bleeding events. This information further complements the results of existing studies, which focused mainly on comparing the efficacy and side effects of warfarin versus DOAC.

To our knowledge, this is the first study that compared QOL and treatment satisfaction in Asian patients on longterm warfarin versus DOAC treatment. Patients who crossed over from warfarin to DOAC or vice versa were excluded in this study to minimize the reporting bias. The analyses of QOL and treatment satisfaction were adjusted for age and treatment duration, which has been highlighted in other studies as important confounding factors. ${ }^{32-34}$ Each domain of SF12 and each item of PACT-Q2 were also analyzed and reported in order to provide a more detailed comparison.
This study had several limitations. First, it was performed in a single center, thus limiting the generalisability of the results. Second, the cross-sectional design might not be able to perfectly reflect the QOL, as QOL may vary over time. Third, the poor physical condition of some patients may have been a deciding factor in the choice of DOAC over warfarin in daily practice. Fourth, the cost of treatment was not taken into account when treatment burden and satisfaction were assessed. Fifth, the amended item B5 of PACT-Q2 for DOAC group was not validated. Sixth, the comparison of item B5 for diet in the warfarin group versus pill intake in the DOAC group may not be fair. Seventh, the reporting of treatment complications was subject to the recall bias of the patients, but this was minimized by double-checking available medical records. A multi-center randomized double-blind study would be the best methodology to eliminate these limitations.

\section{Conclusion}

DOAC is a better option as an oral anticoagulant in patients with NVAF or VTE because of greater satisfaction, more convenience in food intake, less frequent hospitalization, as well as fewer clinically relevant minor bleeds and severe bleeding events. Furthermore, achieving good TTR in patients on warfarin is challenging.

\section{Ethics approval and informed consent}

The UMMC hospital's ethics committee approved this study with reference of Medical Ethic Committee Identity Number (MECID); number 2016823-4147. Written informed consent was obtained from all the study patients.

\section{Abbreviations}

$\mathrm{AF}$, atrial fibrillation; VTE, venous thromboembolism; INR, international normalized ratio; DOAC, direct oral anticoagulants; QOL, quality of life; UMMC, University Malaya Medical Centre; NVAF, non-valvular atrial fibrillation; TTR, time in therapeutic range; SF12v2, Short Form 12v2 Health Survey; PACT-Q2, Perception of Anticoagulation Treatment Questionnaire 2; PF, physical functioning; RP, role physical; BP, bodily pain; GH, general health perceptions; V, vitality; SF, social functioning; RE, role emotional; $\mathrm{MH}$, mental health; PCS, physical component summary; MCS, mental component summary; EQ-5D, EuroQOL instrument; RELY, Randomized Evaluation of 
Long-term Anticoagulant Therapy; EQ-5D-3L, EuroQOL instrument 3 levels; SF36, Short Form-36; ACTS, AntiClot Treatment Scale; XALIA, XA inhibition with rivaroxaban for Long-term and initial anticoagulation in venous thromboembolism.

\section{Acknowledgments}

We want to express our gratitude to all the patients who had participated in the study. This study was fully supported by the Research Acculturation Grant Scheme of the Ministry of Education, Malaysia (RAGS/1/2015/SKK02/ UNIMAS/03/1 and FA052000-0708-0028).

\section{Author contributions}

All the authors contributed substantially to this study, in the following ways: substantial contributions to conception and design, data acquisition, or data analysis and interpretation; drafting the article or critically revising it for important intellectual content; final approval of the version to be published; and agreement to be accountable for all aspects of the work in ensuring that questions related to the accuracy or integrity of the work are appropriately investigated and resolved.

\section{Disclosure}

The authors report no conflicts of interest in this work.

\section{References}

1. Lip GYH, Banerjee A, Boriani G, et al. Antithrombotic therapy for atrial fibrillation. Chest. 2018;154(5):1121-1201. doi:10.1016/j. chest.2018.07.040

2. Kearon C, Akl EA, Ornelas J, et al. Antithrombotic therapy for VTE disease. Chest. 2016;149(2):315-352. doi:10.1016/j.chest.2015. 11.026

3. Holbrook AM, Pereira JA, Labiris R, et al. Systematic overview of warfarin and its drug and food interactions. Arch Intern Med. 2005;165 (10):1095-1106. doi:10.1001/archinte.165.10.1095

4. Wadelius M, Chen LY, Eriksson N, et al. Association of warfarin dose with genes involved in its action and metabolism. Hum Genet. 2007;121(1):23-34. doi:10.1007/s00439-006-0260-8

5. Czuprynska J, Patel JP, Arya R. Current challenges and future prospects in oral anticoagulant therapy. Br J Haematol. 2017;178(6):838851. doi:10.1111/bjh.14714

6. Baglin T. Clinical use of new oral anticoagulant drugs: dabigatran and rivaroxaban. $B r J$ Haematol. 2013;163(2):160-167. doi:10.1111/ bjh. 12502

7. Ware JE Jr., Sherbourne CD, The MOS. 36-item short-form health survey (SF-36). I. Conceptual framework and item selection. Med Care. 1992;30(6):473-483.

8. Keita I, Aubin-Auger I, Lalanne C, et al. Assessment of quality of life, satisfaction with anticoagulation therapy, and adherence to treatment in patients receiving long-course vitamin $\mathrm{K}$ antagonists or direct oral anticoagulants for venous thromboembolism. Patient Prefer Adherence. 2017;11:1625-1634. doi:10.2147/PPA.S131157
9. Wang Y, Kong MC, Lee LH, Ng HJ, Ko Y. Knowledge, satisfaction, and concerns regarding warfarin therapy and their association with warfarin adherence and anticoagulation control. Thromb Res. 2014;133(4):550-554. doi:10.1016/j.thromres.2014. 01.002

10. Weaver M, Patrick DL, Markson LE, Martin D, Frederic I, Berger M. Issues in the measurement of satisfaction with treatment. $\mathrm{Am} \mathrm{J}$ Manag Care. 1997;3(4):579-594.

11. Kravitz RL. Patients' expectations for medical care: an expanded formulation based on review of the literature. Med Care Res Rev. 1996;53(1):3-27. doi:10.1177/107755879605300101

12. Rosendaal FR, Cannegieter SC, van der Meer FJ, Briet E. A method to determine the optimal intensity of oral anticoagulant therapy. Thromb Haemost. 1993;69(3):236-239.

13. Connolly SJ, Pogue J, Eikelboom J, et al. Benefit of oral anticoagulant over antiplatelet therapy in atrial fibrillation depends on the quality of international normalized ratio control achieved by centers and countries as measured by time in therapeutic range. Circulation. 2008;118(20):2029-2037. doi:10.1161/CIRCULATIONAHA.107. 750000

14. Fong DYT, Lam CLK, Mak KK, et al. The short form- 12 health survey was a valid instrument in Chinese adolescents. J Clin Epidemiol. 2010;63(9):1020-1029. doi:10.1016/j.jclinepi.2009. 11.011

15. Mohamed S, Razak TA, Hashim R. Translation, validation and psychometric properties of Bahasa Malaysia version of the Perception of Anticoagulant Therapy Questionnaire (PACTQ). Asian Journal of Biomedical and Pharmaceutical Sciences. 2015;5(48):18-22.

16. Noor NM, Aziz AA. Validity and reliability of the malay version of 12-item short form health survey among postpartum mothers. Malaysian J Public Health Med. 2014;14:1-11.

17. Prins MH, Marrel A, Carita P, et al. Multinational development of a questionnaire assessing patient satisfaction with anticoagulant treatment: the 'Perception of Anticoagulant Treatment Questionnaire' (PACT-Q). Health Qual Life Outcomes. 2009;7:9. doi:10.1186/ 1477-7525-7-9

18. Prins MH, Guillemin I, Gilet H, et al. Scoring and psychometric validation of the Perception of Anticoagulant Treatment Questionnaire (PACT-Q). Health Qual Life Outcomes. 2009;7:30. doi:10.1186/1477-7525-7-30

19. Benzimra M, Bonnamour B, Duracinsky M, et al. Real-life experience of quality of life, treatment satisfaction, and adherence in patients receiving oral anticoagulants for atrial fibrillation. Patient Prefer Adherence. 2018;12:79-87. doi:10.2147/PPA. S131158

20. Stampfuss J, Kubitza D, Becka M, Mueck W. The effect of food on the absorption and pharmacokinetics of rivaroxaban. Int J Clin Pharmacol Ther. 2013;51(7):549-561. doi:10.5414/CP201812

21. Schulman S, Kearon C. Definition of major bleeding in clinical investigations of antihemostatic medicinal products in non-surgical patients. J Thromb Haemost. 2005;3(4):692-694. doi:10.1111/j.15387836.2005.01204.x

22. Kaatz S, Ahmad D, Spyropoulos AC, Schulman S. Definition of clinically relevant non-major bleeding in studies of anticoagulants in atrial fibrillation and venous thromboembolic disease in nonsurgical patients: communication from the SSC of the ISTH. $J$ Thromb Haemost. 2015;13(11):2119-2126.

23. Monz BU, Connolly SJ, Korhonen M, Noack H, Pooley J. Assessing the impact of dabigatran and warfarin on health-related quality of life: results from an RE-LY sub-study. Int $J$ Cardiol. 2013;168 (3):2540-2547. doi:10.1016/j.ijcard.2013.03.059

24. Contreras Muruaga MDM, Vivancos J, Reig G, et al. Satisfaction, quality of life and perception of patients regarding burdens and benefits of vitamin $\mathrm{K}$ antagonists compared with direct oral anticoagulants in patients with nonvalvular atrial fibrillation. $J$ Comp Eff Res. 2017;6(4):303-312. doi:10.2217/cer-2016-0078 
25. Alegret JM, Vinolas X, Arias MA, et al. New oral anticoagulants vs vitamin $\mathrm{K}$ antagonists: benefits for health-related quality of life in patients with atrial fibrillation. Int J Med Sci. 2014;11(7):680-684. doi:10.7150/ijms.8916

26. Marvig CL, Verhoef TI, de Boer A, et al. Quality of life in patients with venous thromboembolism and atrial fibrillation treated with coumarin anticoagulants. Thromb Res. 2015;136(1):69-75. doi:10.1016/j.thromres.2015.04.026

27. Lancaster TR, Singer DE, Sheehan MA, et al. The impact of longterm warfarin therapy on quality of life. Evidence from a randomized trial. Boston Area Anticoagulation Trial for Atrial Fibrillation Investigators. Arch Intern Med. 1991;151(10):1944-1949.

28. Balci KG, Balci MM, Canpolat U, et al. Comparison of health-related quality of life among patients using novel oral anticoagulants or warfarin for non-valvular atrial fibrillation. Anatolian J Cardiol. 2016;16(7):474-481. doi:10.5152/AnatolJCardiol.2015.6334

29. Prins $\mathrm{MH}$, Bamber L, Cano SJ, et al. Patient-reported treatment satisfaction with oral rivaroxaban versus standard therapy in the treatment of pulmonary embolism; results from the EINSTEIN PE trial. Thromb Res. 2015;135(2):281-288. doi:10.1016/j.thromres. 2014.11 .008
30. Cano S, Mantovani L, Folkerts K, et al. Patient-reported treatment experience with oral rivaroxaban: results from the noninterventional XALIA study of deep-vein thrombosis. TH Open. 2018;02(02):e139e146. doi:10.1055/s-0038-1641679

31. Willich SN, Bauersachs R, Gitt AK, et al. Treatment satisfaction in patients with venous thromboembolism as measured with pact-Q2: prefer in vte registry. Value Health. 2014;17(7):A496-A497. doi:10.1016/j.jval.2014.08.1483

32. Almeida Gde Q, Noblat Lde A, Passos LC, Do Nascimento HF. Quality of life analysis of patients in chronic use of oral anticoagulant: an observational study. Health Qual Life Outcomes. 2011;9:91. doi:10.1186/1477-7525-9-40

33. Gadisseur AP, Kaptein AA, Breukink-Engbers WG, van der Meer FJ, Rosendaal FR. Patient self-management of oral anticoagulant care vs management by specialized anticoagulation clinics: positive effects on quality of life. J Thromb Haemost. 2004;2(4):584-591. doi:10.1111/j.1538-7836.2004.00659.x

34. Casais P, Meschengieser SS, Sanchez-Luceros A, Lazzari MA. Patients' perceptions regarding oral anticoagulation therapy and its effect on quality of life. Curr Med Res Opin. 2005;21(7):1085-1090. doi:10.1185/030079905X50624
Patient Preference and Adherence

\section{Publish your work in this journal}

Patient Preference and Adherence is an international, peer-reviewed, open access journal that focusing on the growing importance of patient preference and adherence throughout the therapeutic continuum. Patient satisfaction, acceptability, quality of life, compliance, persistence and their role in developing new therapeutic modalities and compounds to optimize clinical outcomes for existing disease

\section{Dovepress}

states are major areas of interest for the journal. This journal has been accepted for indexing on PubMed Central. The manuscript management system is completely online and includes a very quick and fair peer-review system, which is all easy to use. Visit http:// www.dovepress.com/testimonials.php to read real quotes from published authors. 